\title{
Biomarkers predictive value in early stage non-small cell lung cancer
}

\author{
Umberto Malapelle, Elena Vigliar, Giancarlo Troncone \\ Department of Public Health, University of Naples Federico II, Naples, Italy \\ Correspondence to: Giancarlo Troncone. Department of Public Health, University of Naples Federico II, Via Sergio Pansini 5, 80131 Naples, Italy. \\ Email: giancarlo.troncone@unina.it. \\ Provenance and Peer Review: This article was commissioned by the editorial office, Translational Lung Cancer Research. The article did not undergo \\ external peer review. \\ Comment on: Tu H, Wu M, Huang W, et al. Screening of potential biomarkers and their predictive value in early stage non-small cell lung cancer: a \\ bioinformatics analysis. Transl Lung Cancer Res 2019;8:797-807.
}

Submitted Mar 14, 2020. Accepted for publication Mar 26, 2020.

doi: $10.21037 /$ tlcr.2020.04.16

View this article at: http://dx.doi.org/10.21037/tlcr.2020.04.16

Lung cancer is the principal cause of cancer death worldwide (1). The vast majority of non-small cell lung cancer (NSCLC), diagnosed in advanced stages of disease, has a poor prognosis (2). To improve patient outcome thanks to targeted therapies, assessment of several clinical relevant predictive biomarkers is key (3). In advanced stages of NSCLC, the College of American Pathologists (CAP), the International Association for the Study of Lung Cancer (IASLC) and the Association for Molecular Pathology (AMP) identified panels of "must test genes" and "should test genes" (4) to be assessed by next generation sequencing (NGS) (5). Conversely, in early NSCLC stages no clear recommendations have been issued. To date, in this setting, only serum cancer related biomarkers [e.g., carcinoembryonic antigen (CEA)] have been proposed. While, these latter may somehow be useful predict response to chemotherapy and prognosis (6), they have low sensitivity and specificity, as diagnostic markers (7). Screening by lowdose spiral computed tomography (CT), has recently been proposed by Humphrey et al., who reported in high-risk persons only a $20 \%$ mortality rate reduction (8). Therefore, the unmeet need of molecular markers as diagnostic tool for lung cancer detection in clinical practice makes relevant the study published in this Translation Lung Cancer Research issue by Tu et al. They adopted well-established bioinformatic tools to identify potential biomarkers for the early diagnosis of NSCLC (9). In particular, the Authors interrogated the Gene Expression Omnibus (GEO) database to select the mRNA profiles of differentially expressed genes (DEGs) between tumoral and non tumoral patient samples. Three different gene expression profiles were taken into account: GSE19188, generated from 91 early NSCLC and 65 normal samples from 156 patients; GSE33532, derived from 80 early NSCLC and 20 normal specimens from 20 patients and GSE44077, consisting of 55 early NSCLC and 66 normal samples from 20 patients. On the whole, 221 DEGs were selected, excluding the DEGs overlaps of the three databases. As far as gene expression among DEGs is concerned, 60 genes evidenced an upregulation, whereas 161 were down-expressed in early stage NSCLC respect to normal samples (9). Subsequently, a pathway reconstruction analysis was performed by using the Database for Annotation, Visualization and Integrated Discovery (DAVID). On the overall, by interrogating Gene oncology (GO) and Kyoto Enciclopedia of Genes and Genomes (KEGG) databases the up-regulated genes were principal involved in the mitotic nuclear and cell division, and cell proliferation; whereas genes within the down-regulated group determined signal transduction, angiogenesis, and cell adhesion (Figure 1). Additionally, the Authors carried out Protein-protein interaction (PPI) network of the selected DEGs in order to identify the interaction among proteins. From a prognostic point of view, the Authors underlined the correlation among high mRNA level of ubiquitin-conjugating enzyme E2T (UBE2T), UNF2, cyclin-dependent kinase inhibitor 3 
A

JAK-STAT SIGNALING PATHWAY
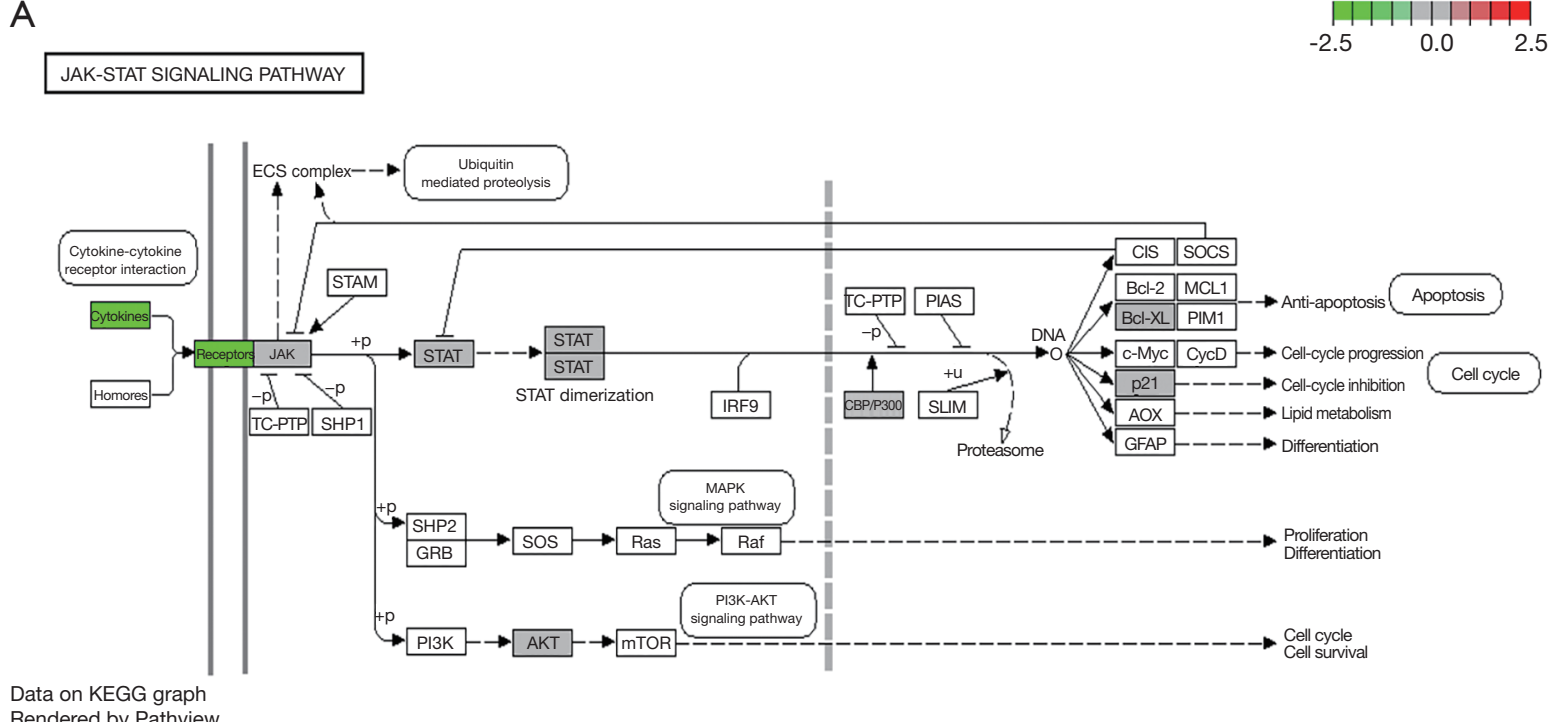

Rendered by Pathview

B
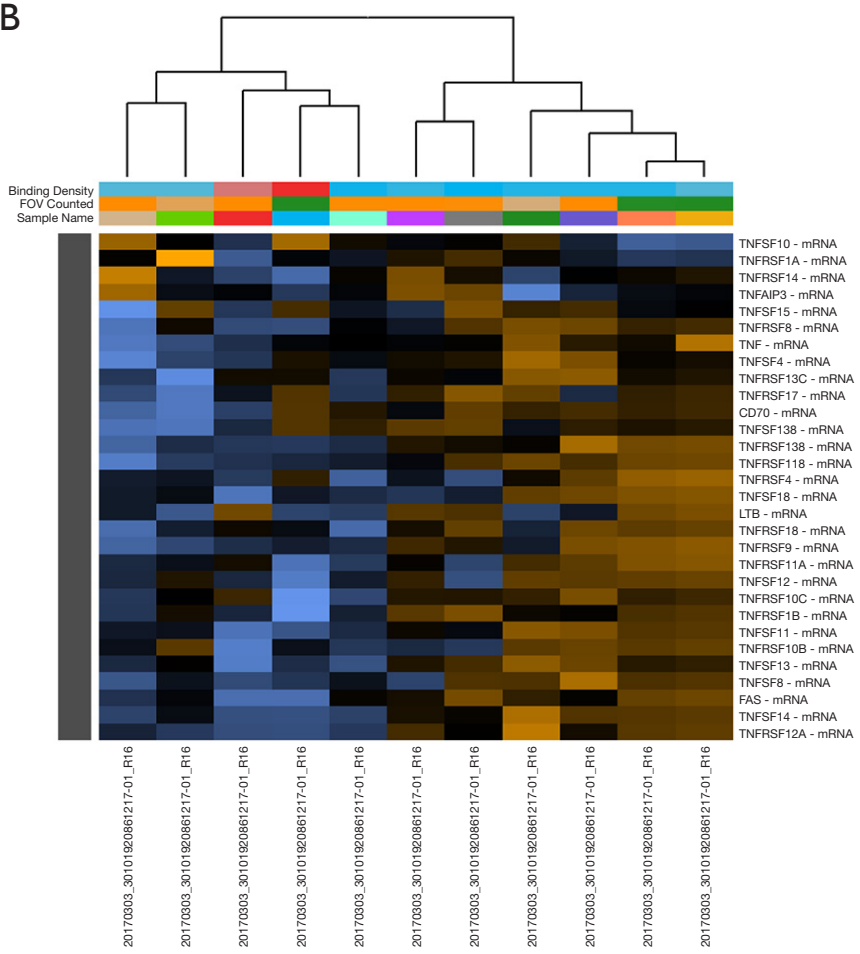

Figure 1 Exemplificative visualization of a pathway reconstruction based on Kyoto Enciclopedia of Genes and Genomes (KEGG) graph built on gene expression data obtained by n-Counter (Nanostring) and analyzed with n-Solver Software 4.0 at the Predictive Molecular Pathology Lab, Department of Public Health of University Federico II of Naples. 
$(C D K N 3)$, anillin $(A N L N)$, Cyclin $\mathrm{B} 2$ (CCNB2), and Cytoskeleton Associated Protein 2 Like (CKAP2L) genes and an overall lower NSCLC patients survival rate (9). The Authors specifically pinpointed the potential role of these genes as predictive biomarkers for the early detection of NSCLC. Moreover, UBE2T plays an important role in the activation of PI3K/Akt pathway, and its upregulation was associated with tumor progression, invasion and metastatization of different cancer types, including NSCLC (10). In addition, high expression levels of UBE2T were related to disease recurrence after surgery (10). $C D K N 3$ was associated with the dephosphorilation of CDK2, and its high expression is a negative prognostic marker in lung adenocarcinoma (11). ANLN showed an important role in cancer development and progression. An in vitro study on NSCLC cells underlined that the down-regulation of $A N L N$ reduced dramatically cell proliferation (9). CCNB2 is associated with the G2/M transition during the cell cycle (12). It has been reported the association between the increasing level of CCNB2 protein expression and poor outcome in NSCLC patients (13). Conversely, the role of UNF2 and CKAP2L in NSCLC still remain unknown, need to be deeply investigated.

On the overall, the paper by Tu et al. strongly emphasized the role of DEGs not only as prognostic but also as potential useful targets for the early diagnosis and treatment of NSCLC patients (9). They highlighted the central role of comprehensive bioinformatics analysis for biomarker screening, as a preliminary step to plan targeted in vivo validation studies. Despite the established role of most of the identified DEGs in crucial stages of cancer development and metastatization, several aspects, as suggested also by the Authors, remain to be further addressed. In fact, the mechanisms of action of some of the genes is not still understood and further knowledge is required to conceivably screen patients in the early stage of disease. Since screening NSCLC by invasive tissue based biomarkers analysis is unfeasible, it would be very interesting in further studies to assess the concordance rate between the data obtained on tissue and paired liquid biopsy. These evidences concur with those generated by a prospective study by Chen $e t$ al., who in early stage disease employed a targeted NGS approach to evaluate the presence of plasma circulating tumor DNA (ctDNA) as a predictive biomarker (14). On the overall, these Authors showed that the vast majority of early stage NSCLC patients (89.7\%) had ctDNA that was quantifiable and potentially testable (14) to detect cancer in asymptomatic high risk patients (14). An integrated radiological and liquid biopsy approach may be a valid tool to identify early stages NSCLC with a significant reduction of costs for the healthcare system and discomfort to the patients (15). A limit of this approach may be related to the low concentration of ctDNA in the early stage of disease; this latter would be overcome by combining DNA, RNA and methylation based analysis (16).

In conclusion, the paper by Tu et al. represents an important contribution in the field of biomarkers role in early stage NSCLC. To favor implementation in the clinical setting, prospective evaluation in randomized trials and matched analysis of tissue and plasma samples are required.

\section{Acknowledgments}

Funding: None.

\section{Footnote}

Conflicts of Interest: All authors have completed the ICMJE uniform disclosure form (available at http://dx.doi. org/10.21037/tlcr.2020.04.16). UM reports personal fees (for service in the speaker bureau and as an advisor) from Boehringer Ingelheim, AstraZeneca, Roche, MSD, Amgen, Merck, outside the submitted work. GT reports personal fees (for service in the speaker bureau and as an advisor) from Roche, MSD, Pfizer, Bayer outside the submitted work. EV has no conflicts of interest to declare.

Ethical Statement: The authors are accountable for all aspects of the work in ensuring that questions related to the accuracy or integrity of any part of the work are appropriately investigated and resolved.

Open Access Statement: This is an Open Access article distributed in accordance with the Creative Commons Attribution-NonCommercial-NoDerivs 4.0 International License (CC BY-NC-ND 4.0), which permits the noncommercial replication and distribution of the article with the strict proviso that no changes or edits are made and the original work is properly cited (including links to both the formal publication through the relevant DOI and the license). See: https://creativecommons.org/licenses/by-nc-nd/4.0/.

\section{References}

1. Siegel RL, Miller KD, Jemal A. Cancer statistics, 2020. 
CA Cancer J Clin 2020;70:7-30.

2. Molina JR, Yang P, Cassivi SD, et al. Non-small cell lung cancer: epidemiology, risk factors, treatment, and survivorship. Mayo Clin Proc 2008;83:584-94.

3. Pisapia P, Pepe F, Troncone G, et al. Predictive biomarkers for molecular pathology in lung cancer. Biomark Med 2020;14:253-7.

4. Lindeman NI, Cagle PT, Aisner DL, et al. Updated Molecular Testing Guideline for the Selection of Lung Cancer Patients for Treatment With Targeted Tyrosine Kinase Inhibitors: Guideline From the College of American Pathologists, the International Association for the Study of Lung Cancer, and the Association for Molecular Pathology. J Thorac Oncol 2018;13:323-58.

5. Vigliar E, Malapelle U, de Luca C, et al. Challenges and opportunities of next-generation sequencing: a cytopathologist's perspective. Cytopathology 2015;26:271-83.

6. Arrieta O, Villarreal-Garza C, Martinez-Barrera L, et al. Usefulness of serum carcinoembryonic antigen (CEA) in evaluating response to chemotherapy in patients with advanced non small-cell lung cancer: a prospective cohort study. BMC Cancer 2013;13:254.

7. Alsabti EA, Kamel A. Carcinoembryonic antigen (CEA) in patients with malignant and non-malignant diseases. Neoplasma 1979;26:603-9.

8. Humphrey LL, Deffebach M, Pappas M, et al. Screening for lung cancer with low-dose computed tomography: a

Cite this article as: Malapelle U, Vigliar E, Troncone G. Biomarkers predictive value in early stage non-small cell lung cancer. Transl Lung Cancer Res 2020;9(4):956-959. doi: 10.21037/ tlcr.2020.04.16 systematic review to update the US Preventive services task force recommendation. Ann Intern Med 2013;159:411-20.

9. Tu $\mathrm{H}, \mathrm{Wu} M$, Huang $\mathrm{W}$, et al. Screening of potential biomarkers and their predictive value in early stage nonsmall cell lung cancer: a bioinformatics analysis. Transl Lung Cancer Res 2019;8:797-807.

10. Perez-Peña J, Corrales-Sanchez V, Amir E, et al. Ubiquitin-conjugating enzyme E2T (UBE2T) and denticleless protein homolog (DTL) are linked to poor outcome in breast and lung cancers. Sci Rep 2017;7:17530.

11. Zang X, Chen M, Zhou Y, et al. Identifying CDKN3 Gene Expression as a Prognostic Biomarker in Lung Adenocarcinoma via Meta-analysis. Cancer Inform 2015;14:183-91.

12. Nam HJ, van Deursen JM. Cyclin B2 and p53 control proper timing of centrosome separation. Nat Cell Biol 2014;16:538-49.

13. Qian X, Song X, He Y, et al. CCNB2 overexpression is a poor prognostic biomarker in Chinese NSCLC patients. Biomed Pharmacother 2015;74:222-7.

14. Chen KZ, Lou F, Yang F, et al. Circulating Tumor DNA Detection in Early-Stage Non-Small Cell Lung Cancer Patients by Targeted Sequencing. Sci Rep 2016;6:31985.

15. Liang $W$, Zhao Y, Huang W, Liang H, Zeng H, He J. Liquid biopsy for early stage lung cancer. J Thorac Dis. 2018;10:S876-81.

16. Pisapia P, Malapelle U, Troncone G. Liquid Biopsy and Lung Cancer. Acta Cytol 2019;63:489-96. 